\title{
Topological Metal-Insulator Transition in Narrow Graphene
}

\section{Nanoribbons?}

Aristides D. Zdetsis*1,2 and E. N. Economou ${ }^{2}$

${ }^{1}$ Molecular Engineering Laboratory, Department of Physics, University of Patras, Patras 26500 GR, Greece

${ }^{2}$ Institute of Electronic Structure and Laser, Foundation for Research \&Technology Hellas, Vassilika Vouton, P.O. Box 1385, Heraklion, Crete GR-71110, Greece and Department of Physics, University of Crete

\begin{abstract}
We show that very narrow armchair graphene nanoribbons (AGNRs) of length L and width of 2 zigzag-rings undergo a metal-insulator-like transition at a critical length $L_{\mathrm{c}} \approx 10 \mathrm{~nm}$, where the energy gap drops rather abruptly, and the conductivity, estimated, through an invoked computational scheme, rises almost discontinuously to a value of $\sim 7 \frac{e^{2}}{h}$, between $10 \frac{e^{2}}{h}$ for a perfect quasi one-dimensional system, and the nominal minimum conductivity of graphene, $\sigma_{\min }=4 \frac{e^{2}}{h}$. At $L_{c}$, the aromatic and non-aromatic rings are interchanged, and sharp peaks appear in the density of states around the Fermi level, suggesting metallic-like behaviour. Such peaks linked to edge states at the Dirac point(s) coincide with the charge-neutrality point(s), associated with $\sigma_{\min }$. Thus, we have an uncommon combination of interrelated "short-long", “core-edge," topological-aromatic transition(s) due to strong quantum confinement, driven by inversion symmetry conflict. The bandgap decreases as $\mathrm{L}^{-2 / 3}$, for $\mathrm{L}<L_{c}$ and $\ln \left(\mathrm{L}-L_{c}\right)$ for $\mathrm{L}>L_{c}$. These effects are practically non-existent for wider AGNRs.

*zdetsis@upatras.gr
\end{abstract}


With the usual top down procedure for obtaining graphene nanoribbons (GNRs), for the purpose of opening a (possibly adjustable) bandgap in graphene, the GNRs obtained are not structurally pure in the sense that they could not be separated in structurally pure forms like fullerenes for example. ${ }^{1}$ This was a rather serious drawback for their full understanding and the precise design of GNRs with tunable gaps, until the latest developments of atomically precise GNRs, and in particular armchair GNRs (AGNRs), synthesized by bottom-up processes using molecular precursors, which revolutionized the field of AGNRs. ${ }^{2-7}$ At the same time their complete structural and electronic characterization was facilitated by using scanning tunneling microscopy (STM) and spectroscopy (STS),${ }^{1-7}$ not always unambiguously, without discrepancies ${ }^{8}$, as could be expected for such new field. The two key reasons for such discrepancies were recognized by the present authors ${ }^{8}$ to be the finite length of the bottom-up fabricated AGNRs, and the (seemingly unrelated) edge states $^{6-7}$ at their finite zigzag edges. It is demonstrated hereby that these two interrelated aspects (edge states, finite length) are not accidental, but fundamental properties deeply rooted in the molecular (chemical), physical, and topological properties of graphene and graphene-based structures (as the present AGNRs). These key properties are shown to lead to new and novel phenomena, such as an apparently quasi-“phasetransition(s)" at a critical length $\mathrm{L}_{\mathrm{c}}$ involving in a coordinated way transport, electronic, aromatic, and topological aspects in ultra-narrow AGNRs and in the region of the corresponding Dirac points (DP); the latter in AGNRs have been associated with edge states and sharp peaks in the vicinity of the Fermi level. ${ }^{9-10}$ These peaks often create an artificial maximum in the "simulated" density of states (DOS) around and at the Fermi level, $\operatorname{DOS}\left(\mathrm{E}_{\mathrm{f}}\right)$, (see text below Fig.2) suggesting a tendency towards metallic behavior for the ultrathin AGNRs examined here, and therefore one 
could considered such transition at $\mathrm{L}_{\mathrm{c}}$ as an insulator-metal transition, in view also of the abrupt drop of the energy gap and the almost discontinuity in the conductivity at this length, as is shown in Fig. 1 below. Moreover, DPs, in the absence of disorder, are known to coincide with the charge-neutrality points (CNP), related to the minimum conductivity of graphene. ${ }^{11-13}$ Thus, one could also assume that such transition is related to a transition from below to above the minimum conductivity, which by itself is a very interesting and controversial phenomenon, especially for its exact value. ${ }^{12-13}$ On top of this, aromaticity and symmetry (inversion symmetry in particular) are the hidden but fundamental key quantities for the electronic behavior at this region of DP (and, therefore $\mathrm{CNP})^{9-10}$, suggesting that we have an aromatic or topological transition. In what follows these alternative interpretations and questions will be discussed and rationalized, if possible, starting with the results of Fig.1. Parenthetically, we should emphasize that it is a very important and happy coincidence that such ultra-narrow AGNRs have been already synthesized (by two different groups) with modern bottom-up approaches, ${ }^{3-4}$ and therefore the present results could in principle be tested experimentally. For the common top-down manufactured AGNRs the (finite) length (and the resulting zigzag edge states) are rather irrelevant (been theoretically infinite), with all properties characterized solely by the AGNR's width $\mathrm{N}$, which is usually measured by the number of the carbon atoms across the AGNR's physical width (which is equal to the number of carboncarbon dimer lines making up their width). Alternatively, the width can be also described by the number $\mathrm{Z}$ of the zigzag edge rings, $\mathrm{N}=2 \mathrm{Z}+1$. The description in terms of $\mathrm{Z}$ (rather than $\mathrm{N}$ ) is more "natural" and direct ${ }^{9-10}$, as will become clear below. To assess the data for the possible metal-insulator (or rather insulator-metal) transition in the $\mathrm{Z}=2(\mathrm{~N}=5)$ AGNR, we have plotted in terms of L in Fig.1 the key parameters 
for such transition which are: $1^{\text {st }}$ ) the energy gap between the highest occupied and lowest unoccupied molecular orbital (MO), HOMO and HOMO-LUMO respectively; and $2^{\text {nd }}$ ) the conductivity (or a very good estimate of it as will be described below).
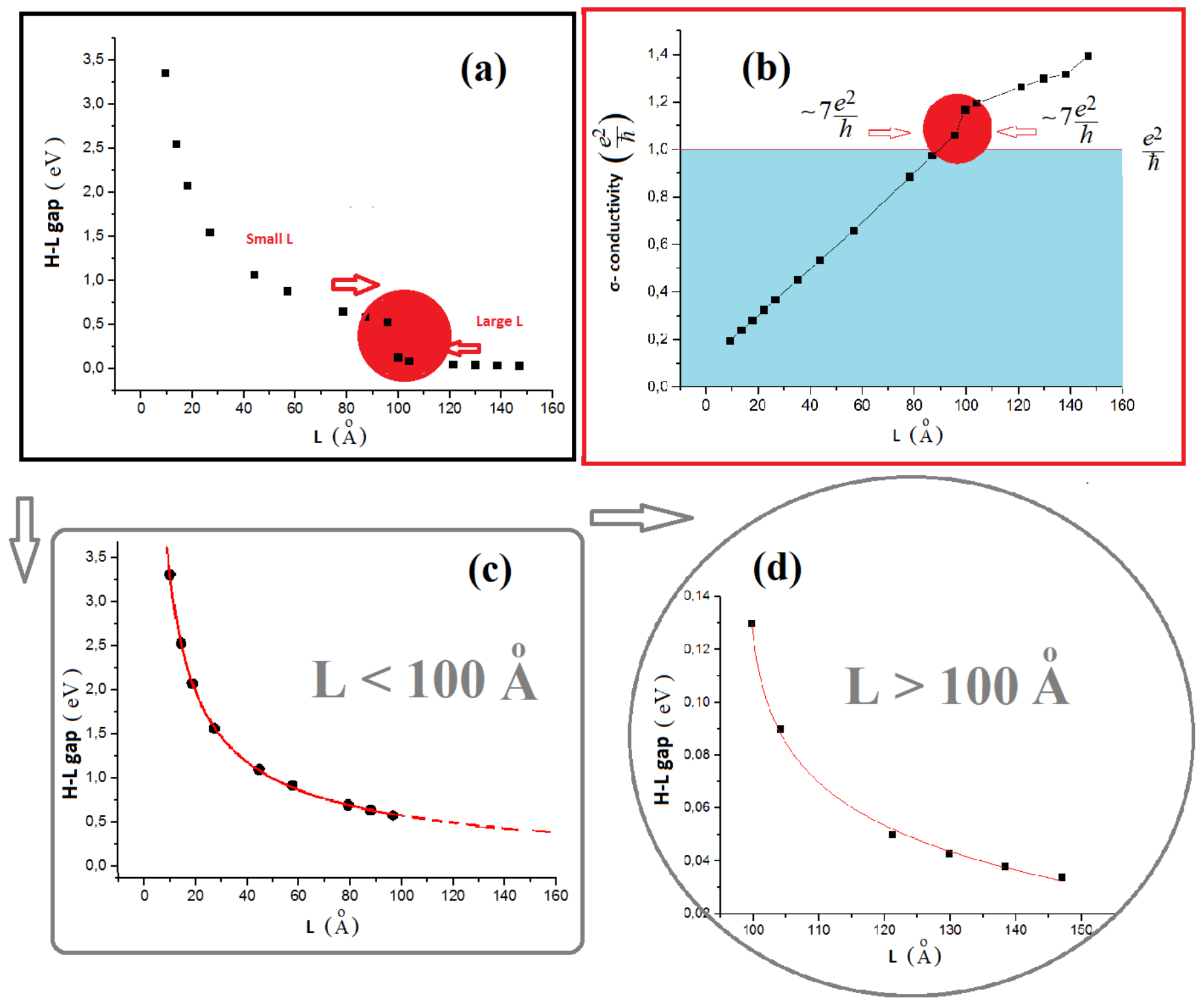

FIGURE 1. Electronic properties of the $\mathrm{Z}=2(\mathrm{~N}=5)$ wide $A G N R$ as a function of length: (a) HOMO-LUMO gap (in $\mathrm{eV}$ ) as a function of length. The discontinuity around $\mathrm{L}=100 \AA$, separating "small L" from "large L" is designated by a red circle; (b) The L-dependence of the conductivity (see text) in atomic units $\frac{e^{2}}{\hbar}\left(=2 \pi \frac{e^{2}}{h}\right)$. The region of the critical length is indicated by a red circle, and the region below the value 
$1 \frac{e^{2}}{\hbar}$ is highlighted light blue, while the critical value of $\sim 7 \frac{e^{2}}{h}$ is shown with red arrows; (c) and (d) Non-linear fits of the L-dependence of the HOMO-LUMO gap in

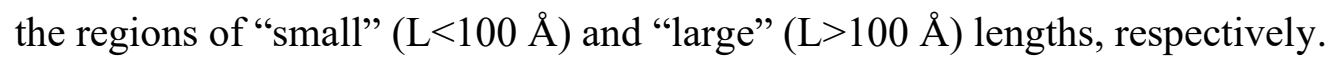

In Fig. 1(a) the calculated HOMO-LUMO gaps, $\Delta \mathrm{E}_{\mathrm{g}}$, are shown point by point. Around $L=100 \AA$ a discontinuity of the gap (to $1 / 4$ of its value) is clearly evident implying a tendency towards insulator-metal transition. To describe the transition and find the possible critical length $\mathrm{L}_{\mathrm{c}}$, several non-linear least square fits of polyonymic, exponential and logarithmic forms were performed separately for "small" L and "large" L regions. The best fit for the small L region, is of a power law form $\Delta E_{g} \sim$ $L^{-0.666} \sim L^{-\frac{2}{3}}$, which is shown in Fig. 1(c); whereas for the "large" L region" the best possible fit obtained, was of the logarithmic form $\Delta E_{g} \sim \ln [L-(98.7 \pm 0.2)]$, shown in Fig. 1(d), suggesting a critical length $L_{c} \approx 98.7 \pm 0.2 \AA$, taking place between lengths $\mathrm{A}=22$ and $\mathrm{A}=23$ armchair rings (or equivalently between $\mathrm{L}=95.6 \AA$ and $\mathrm{L}=99.7 \AA$ ). The exponent $-\frac{2}{3}$ in the region of the strong quantum confinement could be considered as the analog of the "fractal dimensionality" of this AGNR. Going back to the conductivity, shown in Fig. 1(b) we need to describe briefly the simple, but novel and powerful way employed for its estimate. The conductivity was obtained by dividing the induced by the external electric field zig-zag edge charge $\delta q$ (equal by definition to the ratio of the dipole moment $D$ over the length $L$ of the AGNR, $\delta q=D / L)$, by its width $W$, by a characteristic time $\delta t$, and by the applied electric field. In estimating $\delta t$ one has to distinguish between two rather opposite regimes: the one where the losses (both material and radiation) are heavy and as a result 
equilibrium is established by exponential decay characterized by the relaxation time $\tau$; and the case of almost negligible losses, where equilibrium is established through repeated (but decaying) oscillations. In the latter case, which is probably more appropriate for a graphene ribbon given the very high mobility of graphene, $\delta t$ is expected to be approximately $T / 4$, where $T=2 \pi / \omega ; T$ and $\omega$ are respectively the period and the frequency of these oscillations. The frequency squared is given by the well known formula $\kappa / m$, where $m=N m_{e}$ is the total mass of the $N$ edge electrons and $\kappa$ is the "spring constant" experienced by the number $N$ of the oscillating zigzag edge electrons ( $N=5$ in our case). Their average displacement $x$ is expected to be of the order of Bohr's radius $a_{B}$ and it is connected to the edge charge $\delta q$ by the relations $\delta q=N \times e \times\left(x / a_{B}\right)$. The "spring constant" $\kappa$ is given by the force $F$ over the displacement $x, \kappa=F / x$; the force $F$ exercised on the $N$ electrons by the external field $\varepsilon$ is $F=N \times e \times \mathcal{E}$. Hence $\kappa=F / x=N e \mathcal{E} /\left(a_{B} \delta q / N e\right)=(N e)^{2} \varepsilon / a_{B} \delta q$. Combining the above relations with the expression or Bohr's radius, $a_{B}=\hbar^{2} / m_{e} e^{2}$ we end up with the following relation for the conductivity $\sigma$ :

$$
\sigma \approx \frac{2}{\pi W}\left(\frac{N \delta q}{\varepsilon}\right)^{1 / 2} \frac{e^{2}}{\hbar}=\frac{2}{\pi W}\left(\frac{N D}{L \varepsilon}\right)^{1 / 2} \frac{e^{2}}{\hbar}
$$

which is producing the graph shown in Fig. 1(b). It must be made clear that Eq. (1) is an overestimate of the conductivity within the employed physical rational for two reasons: (1) All losses were omitted. (2) Essentially, an infinity mobility was implicitly assumed by adopting the oscillatory nature of approaching equilibrium; however, near the DPs, there are localized edge states the contribution of which to the mobility is zero. As we can see in Fig. 1(b), the calculated conductivity (which, 
anyway, overestimates the actual value) in the strong quantum confinement region starts from values near zero, growing linearly with $\mathrm{L}$ approaching at $L_{c}$ the critical value $1.16 \frac{e^{2}}{\hbar} \sim 7 \frac{e^{2}}{h}$ which is between the value $10 \frac{e^{2}}{h}$ associated with a perfect quasi-one-dimensional system consisting of five non-interacting channels ${ }^{14}$, and the nominal value $\sigma_{\min } \sim 4 \frac{e^{2}}{h}$ for the minimum conductivity of Graphene ${ }^{11}$, which is obtained at $\mathrm{CNP}(\mathrm{s})$; the latter coincides with the DPs in the absence of disorder. In making these comparisons, one must keep in mind that the accurate value of $10 \frac{e^{2}}{h}$ for quasi-one-dimensional systems is obtained assuming on the one hand a perfect conductor (an assumption overestimating the conductivity) and on the other by including the contact resistance to external leads (an inclusion underestimating the conductivity) ${ }^{\mathbf{1 4}}$. Moreover, several estimates for the minimum conductivity in Graphene exist in literature ranging from 2 to 12 times $e^{2} / h$. The conclusion is that the value obtained in the present work for the conductivity of the $N=5$ AGNR at $L=L_{c}$ is about equal to both the minimum conductivity of Graphene and the conductivity for quasi-one-dimensional systems, given the uncertainties in estimating the conductivity in each one of these systems. This approximate coincidence is surprising and possibly very significant and novel, but no further interpretation or speculation about this could be attempted at this time. It is interesting to observe however at this point that the linear dependence of the conductivity in the strong quantum confinement limit implies according to eq. (1) a growth of the dipole moment proportional to the third power of length, $D \sim L^{3}$ in that region.

Now, as was mentioned earlier, on top of this and at the same time with these changes in the gap and the conductivity, we also have at $L_{c}$ discontinuous changes in the 
aromatic properties and the morphology of the DOS, DOS $\left(E_{f}\right)$, as shown in Fig. 2, which are obviously connected with the ones already presented. This should have been expected, since as was shown in earlier works, ${ }^{9-10}$ aromaticity is the fundamental property and driving force (together with symmetry frustration) for all electronic, aromatic, and structural changes of AGNRs and other graphene-based structures. For AGNRs aromaticity is described by the distribution of aromatic (or "full") and nonaromatic ("empty") rings, known as aromaticity pattern in which the aromatic (full) rings are denoted by a full red (on line) circle at their center.
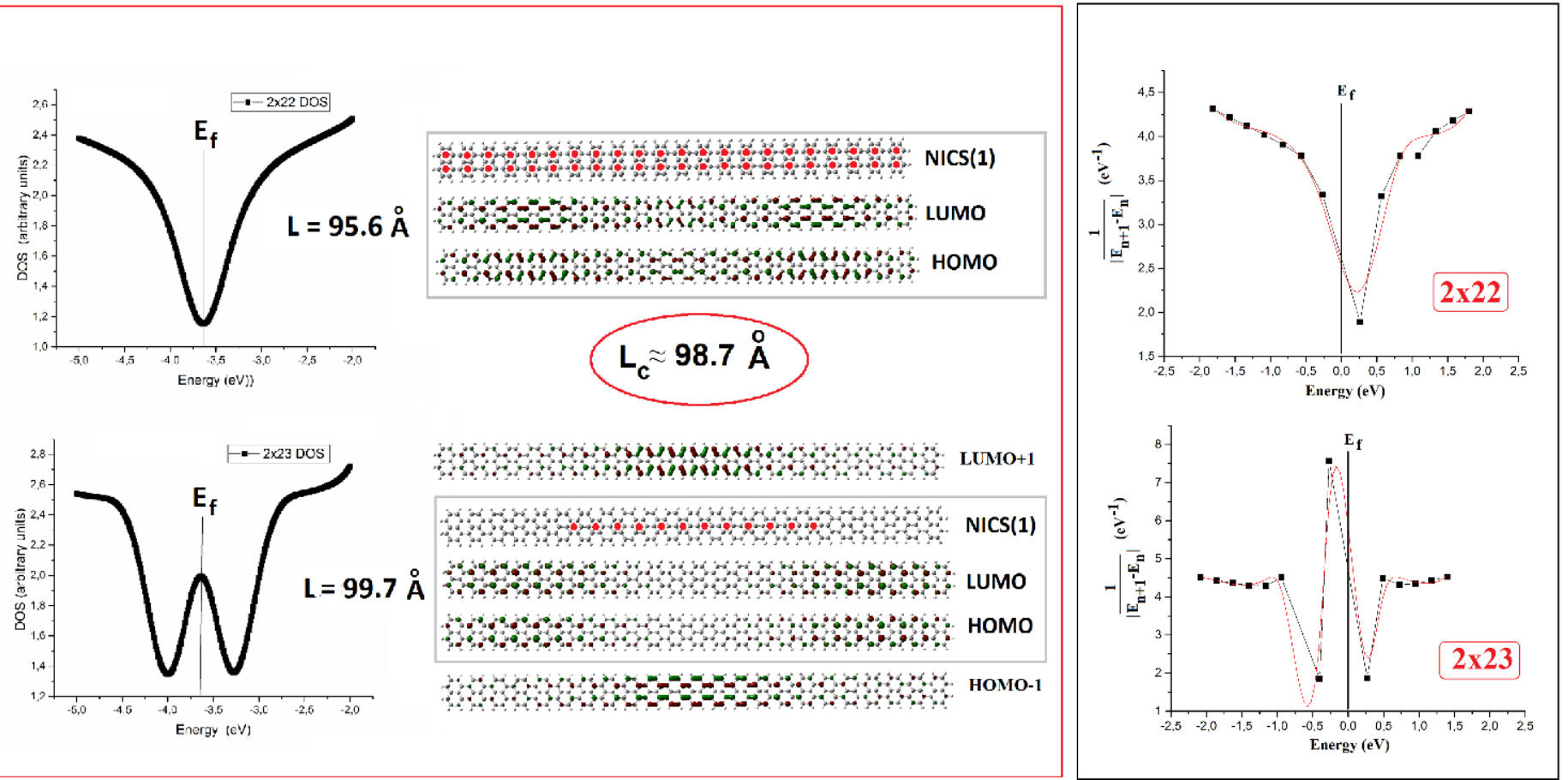

(a)

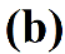

FIGURE 2. (a) Changes in the "simulated" DOS around the Fermi level $\left(\mathrm{E}_{\mathrm{f}}\right)$ of the $\mathrm{Z}=2(\mathrm{~N}=5)$ AGNR for lengths around the critical length $\mathrm{L}=95.6 \AA$ (top) and $\mathrm{L}=99.7 \AA$ (bottom) on the left, together the with corresponding changes in the aromaticity patterns, based on the NICS(1) aromaticity index, ${ }^{15}$ and the HOMO, LUMO orbitals, on the right. For the second (bottom) case the HOMO-1 and LUMO+1 orbitals are also shown for comparison. (b) The corresponding diagrams of $1 /\left|E_{n+1}-E_{n}\right|$ as a 
function of energy $E$ or $\left(E_{n}\right)$, which better describes "DOS" (see text). Red lines (on line) represent cubic spline fits.

To evaluate the (degree of) aromaticity of each ring of the AGNR we adopt the simplest and most widespread aromaticity index of nucleus independent chemical shift (NICS) ${ }^{15}$, evaluated at 1 Ångstrom above the center of the given ring, NICS(1). The index NICS(1), which is based on the magnetic criteria for aromaticity, has been proven adequate for the present (and related) work(s). ${ }^{8-10,15-16}$ We can observe in Fig. 2(a) a change of the aromaticity pattern in such a way that empty rings become aromatic and vice versa. At the same time (same length, $\mathrm{L}_{\mathrm{c}}$ ) the "simulated" DOS at the Fermi level, $\operatorname{DOS}\left(\mathrm{E}_{\mathrm{f}}\right)$, in Fig. 2(a) turns from a local minimum to a local maximum, possibly suggesting an insulator to metal transition. The "simulated" DOS in Fig.2 (a), used for relative comparisons, was obtained by gaussian broadening of the discrete levels by $0.02 \mathrm{eV}$. However, this could be sometimes misleading since strictly speaking DOS is defined for a quasi-continuum spectrum, not discrete as the present. For this reason in Fig. 2(b) we have in parallel plotted the inverse of the level spacing $1 /\left|E_{n+1}-E_{n}\right|$ which for $\mathrm{n} \rightarrow \infty$ approaches the real DOS. The conclusions drawn from both are practically the same. The new (edge) states around the Fermi level (which have been shown to correspond to the Dirac points ${ }^{9-10}$ ) give a local maximum above the minimum of the gap, before the critical Length $\mathrm{L}_{\mathrm{c}} \approx 98.7 \AA$. Thus, at the same time, at the critical length $\left(\mathrm{L}=L_{c}\right)$ we have the appearance of:

$1^{\text {st }}$ ) edge states, which are responsible for the drop in the H-L gap shown in Fig. 1a, and its logarithmic dependence for $L_{c} \leq L$,

$2^{\text {nd }}$ ) DPs,

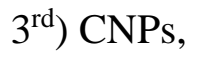


$\left.4^{\text {th }}\right)$ conductivity comparable to the nominal quantum minimum for Graphene, $\sigma_{\min }$, and the one for quasi-one-dimensional systems.

$5^{\text {th }}$ ) interchange between full and empty rings.

Thus, we have an unusual multiple transition. Yet, we have not finished yet.

$\left.6^{\text {th }}\right)$ Such "full-empty" rings transition has been shown earlier ${ }^{9-10,16}$ to be associated with: (6a) a transition from one shell to the next, in the shell "model" 16 ; together with (6b) an interchange between HOMO and LUMO orbitals; and (6c) a change in the inversion symmetry (parity) of the "active" or "effective" HOMO and LUMO orbitals, denoted by an asterisk, $\mathrm{HOMO}^{*}, \mathrm{LUMO}^{*}$. As was pointed out before, the HOMO, LUMO orbitals, appearing only for $L_{c} \leq L$, are associated with the "artificial" edge orbitals . Neglecting these non-representative edge states, the nearest orbitals acting in the present case as the conventional HOMO, LUMO are the HOMO* $\equiv$ HOMO-1 and LUMO* $\equiv$ LUMO+1. In other words, after the "transition", i.e. after the appearance of the edge states, which coincide with the nominal HOMO and LUMO, the HOMO* and LUMO*orbitals (in contrast to the usual HOMO, LUMO) convey and describe the "correct physics" and have well defined invariant symmetry properties, related with the corresponding aromaticity patterns. ${ }^{10}$ In this respect, Fig 1(d), describing the HOMO-LUMO gap after the "transition" is misleading, since the real properties of the AGNR are described by the HOMO*-LUMO*, not the HOMO-LUMO gap. We will come back to this point later below after examining some wider AGNRs. Since edge states and the unavoidable need to introduce effective HOMO*-LUMO* MOs exist for all other (wider) AGNRs of all categories $(\mathrm{Z}=3 \mathrm{n}, 3 \mathrm{n} \pm 1, \mathrm{n}=1,2, \ldots)$; it is natural to search for analogous transitions for them as well. Let us consider first AGNRs of the same type, $Z=3 n-1$, for $n=2$, i.e. $Z=5, N=11$. Obviously, it would be expected that the corresponding 
"metal-insulator" transition, if any, should occur at a smaller critical length, since lateral quantum confinement would be weaker. This is verified emphatically in Fig. 3 , where it is illustrated that such type of transition would occur at lengths between 2 and 3 armchair rings, or between $9.31 \AA$ and $13.45 \AA$; while the corresponding width is $14.13 \AA$ (larger than the length). Comparing with the $6.78 \AA$ width of the $\mathrm{Z}=2$ (N=5) AGNR, we can see that, although the corresponding width is almost doubled, the corresponding "critical" length (about $\approx 11.4 \AA$ ) has been reduced by roughly a factor of $10(\approx 8.8$ exactly $)$, which clearly suggests that quantum confinement is more sensitive to width (with zigzag bonds) than to length (with armchair bonds).

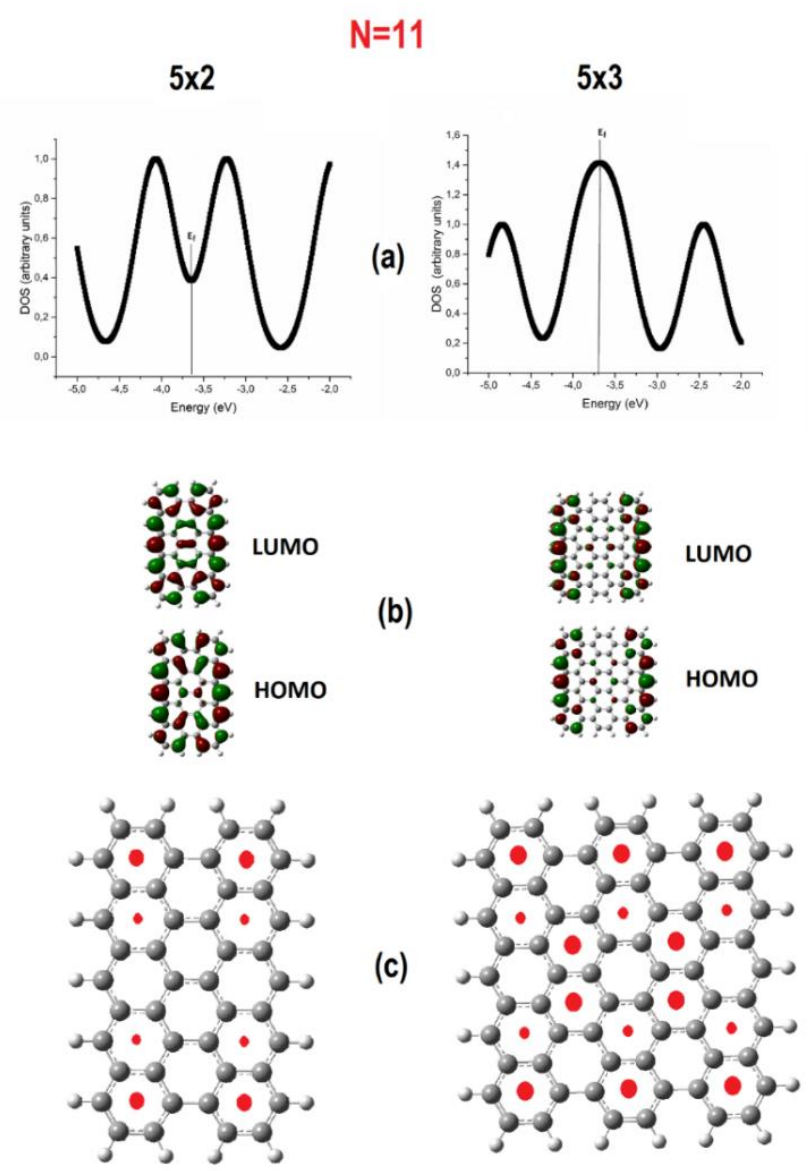

FIGURE 3. The $\mathrm{Z}=5$ (N=11) AGNR before (left) and after (right) "transition": Simulated DOS (a), HOMO, LUMO orbitals (b), and aromaticity patterns (c). The 
size of the dots in the centers of the "aromatic" (or "full") rings is proportional to the magnitude of NICS(1) ${ }^{14}$ aromaticity index.

Nevertheless, since AGNRs of length around $10 \AA$ (and width around $14 \AA$ ) are rather meaningless, such transition is not important or meaningful for these AGNRs. Moreover, we can observe that the aromaticity pattern before and after "transition" is rather irregular, which is due to the non- or mixed aromaticity of the Z=3n-1 AGNRs, in contrast to the ones for $Z=3 n$ and $Z=3 n+1$, which are characterized by "pure" aromaticity patterns similar to coronene (CO) and circumcoronene (CIRCO), respectively. ${ }^{10}$ We should expect that if we want to try to extend this idea to AGNRs of the other families $(Z=3 n$, and $Z=3 n+1, n=1, \ldots)$ we should in principle reach similar (due to quantum confinement), but not identical (due to aromaticity) conclusions. For this purpose, we consider the thinnest $Z=3 n$, and $Z=3 n+1$ AGNRs for $n=1$. As we can see in Fig. 4 in which we have summarized the results, if we want to assign critical lengths for both of them, they should be in the region around 15-20 $\AA$ (between 4-5, and 3-4 armchair rings), which is practically nonsense. However, for these AGNRs, such "transition" should have a very special meaning due to aromaticity. It becomes clear from Fig. 4 that for AGNRs of widths $Z=3$ and $Z=4$, in the region of quantum confinement (in the left part of the figure) the aromatic properties and the characteristic aromaticity patterns ( $\mathrm{CO}$ and $\mathrm{CIRCO})$, together with the topological edge states, have not fully developed, although "aromatic" rings with relatively low aromaticity, based on the aromaticity index NICS(1), are present but in incomplete arrangements, due to spatial restrictions (confinement). Thus, aromaticity patterns (with relatively larger aromaticity indices ${ }^{\mathbf{1 4}}$ ) and topological edge states appear together. 


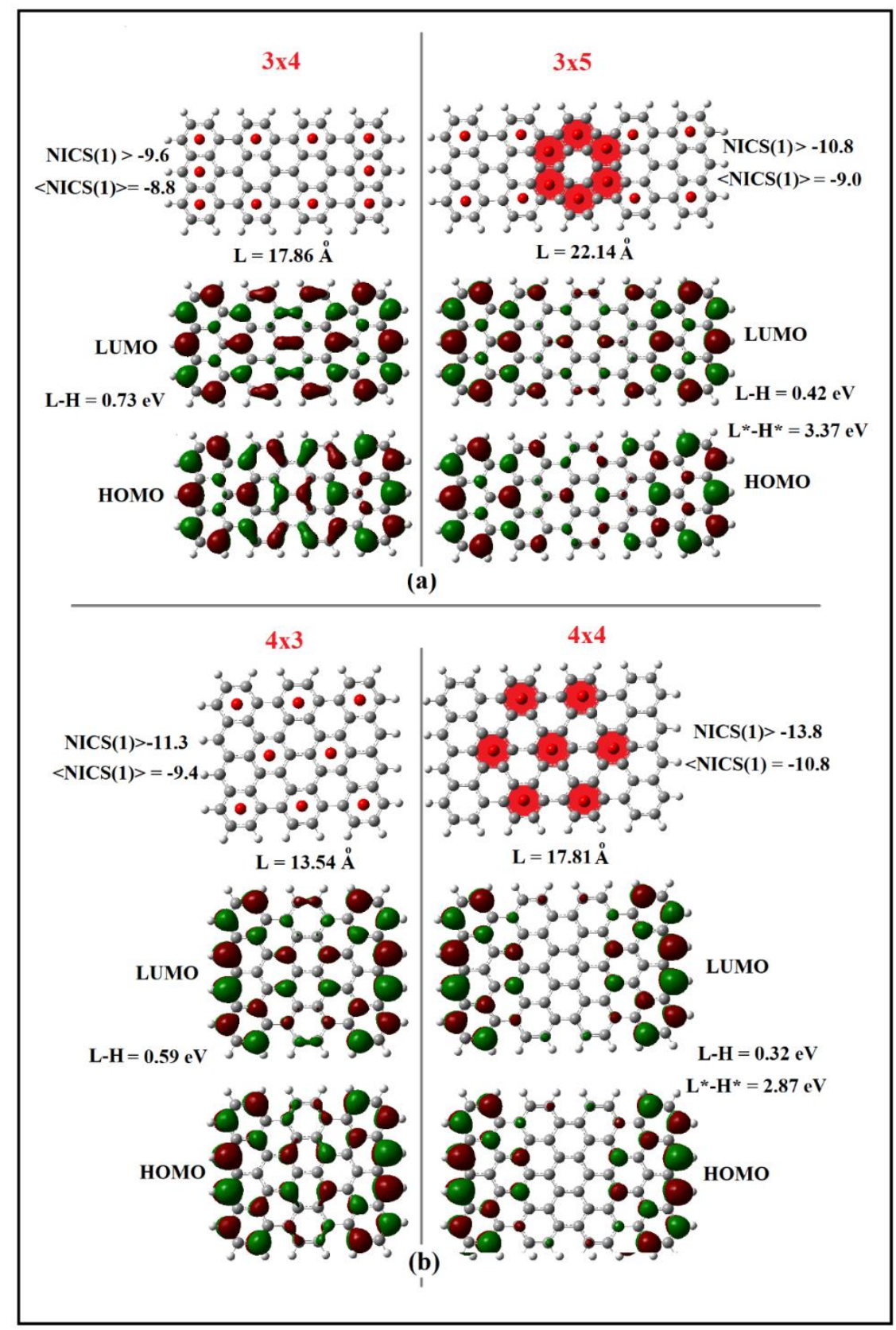

FIGURE 4. The $\mathrm{Z}=3 \mathrm{n}$ (a), and $\mathrm{Z}=3 \mathrm{n}+1$ (b), $\mathrm{n}=1$, AGNRs before (left) and after(right) "transition", illustrating changes in the aromaticity patterns, and the corresponding average and maximum NICS(1) values ( $\langle$ NICS(1)〉 and NICS(1), respectively); together with the HOMO, LUMOs and the corresponding HOMO-LUMO (L-H), and HOMO*-LUMO* $\left(\mathrm{L}^{*}-\mathrm{H}^{*}\right)$ gaps. The full red coloured (online) rings illustrate the characteristic $\mathrm{CO}$ and $\mathrm{CIRCO}$ aromaticity patterns respectively. 
This corroborates previous suggestion(s) ${ }^{9-10}$ that aromaticity (and in particular the occurrence of two, and only two, aromaticity patterns) is a topological property. Moreover, in this case (contrary to the $\mathrm{Z}=3 \mathrm{n}-1$ case with "mixt aromaticity") the contribution (and the significance) of length and width is practically equivalent for quantum confinement. For instance, in terms of the number of rings, we can observe in the left part of Fig.4 that the number of width (zigzag) rings in one AGNR is equal to the number of length (armchair) rings of the other $(3 \times 4=4 \times 3)$. In addition but not independent of this, for these AGNRs the symmetry of the effective HOMO* and LUMO* orbitals is well defined (as the aromaticity) consisting of two types $b_{2 g}$ and $b_{3 u}$ of even and odd parity respectively, which are the $D_{2 h}$ symmetry analogous to the hexagonal $e_{1 g}$ and $e_{2 u}$ HOMO, LUMOs, showing no edge states and fully compatible with the requirements of both, molecular and "sublattice" (or bipartite), symmetry groups $^{9-10}$. In contrast, in the $\mathrm{Z}=2(\mathrm{~N}=5) \mathrm{AGNR}$, which is are characterized by mixt aromatic and topological properties ${ }^{9-10}$, the "frontier" MOs (including HOMO* and LUMO*) in a large region around the Fermi level form an alternating sequence between $a_{u}$ and $b_{1 g}$ symmetries, which are "bulk-forbidden." $9-10$ Therefore, although the origin of the transition in all cases $(Z=3 n, 3 n \pm 1)$ is the same ( quantum confinement), the transition in the very narrow $\mathrm{Z}=2(\mathrm{~N}=5)$ AGNR has different consequences and significance. For this (and only this) AGNR, quantum confinement is anisotropic with respect to width and length, and for this (and only this) AGNR such transition is important and meaningful. For practical purposes, in all other wider AGNRs the edge states can be considered as always present for all (meaningful) lengths. Such states are topologically protected by symmetry. It is suggested here that at the same time such edge states shield to a large degree the "inner" effective $\mathrm{HOMO}^{*}$ and LUMO* states from the influence of the substrate, minimizing the 
substrate contribution to the measured energy gap. This can explain the very good agreement of the HOMO*-LUMO* gap with experimental STS measurements. ${ }^{8}, 10$ The effective HOMO*-LUMO* gap for the $\mathrm{Z}=2(\mathrm{~N}=5)$ AGNR, obtained from the longer AGNRs with length $\mathrm{L}>\mathrm{L}_{\mathrm{c}}$ saturates around $1.2 \mathrm{eV}$, which should be ascribed as the active bandgap. In this respect the effective "bandgap", in contrast to the conventional one shown in Fig. 1, not only is not reduced (towards "more metallic" state), but instead is expanded after the transition and remains practically constant afterwards. In this respect it appears that the $\mathrm{Z}=2(\mathrm{~N}=5)$ AGNR is not really metallic or "near metallic" as it is stated many times in the literature (see for instance ref. 4), and that we do not have in fact a real metal-insulator transition. It is interesting to compare at this point with the higher level LDA+GW results ${ }^{16}$ which predict for this AGNR Z $=2(\mathrm{~N}=5)$ an even larger gap of $1.7 \mathrm{eV}$ (certainly not metallic). If we move towards a larger $n$ value (i.e. $n=2$ ) within the present $Z=3 n-1$ family, we get for $n=2$ (i.e. $\mathrm{Z}=5, \mathrm{~N}=11$ ) a HOMO*-LUMO* gap of $0.7 \mathrm{eV}$, whereas the value obtained by the $\mathrm{LDA}+\mathrm{GW}$ method is $0.9 \mathrm{eV}$, in good agreement (especially if one considers the relative complexity of the two methods). If we compare with experiment, wherever available, the HOMO*-LUMO* value is always much closer (in some cases almost identical) to the experimental results, ${ }^{8,10}$ compared to the LDA+GW results which always give larger values, ${ }^{16}$ which are usually compensated by equally large corrections assigned to AGNR-substrate interactions. Such corrections, which screen the actual values of the gap, are very tricky depending strongly ${ }^{8}$ (among others) on the AGNR-substrate distance. We believe that such interactions should be "small", apparently due to the screening from the "surface" edge-electrons on the "internal" electrons of the HOMO* (LUMO*) states, as was mentioned before. In our earlier work, ${ }^{8}$ we have estimated such corrections for the AGNRs examined here to be of the 
order of $8 \%$, which would practically make no difference whatsoever, especially in view of much larger uncertainties in the literature. ${ }^{3-4,8}$ In any case, this is rather irrelevant for the present results, which also seem to be practically irrelevant for other wider AGNRs. Nevertheless, the implications should be far reaching. The real answer to the question of what kind of transition we have is extremely interesting and multidimensional, involving a combination of interrelated aromatic, electronic, transport, and topological properties together with several rather controversial (but extremely interesting) concepts and results, such as edge states, minimum conductivity, and aromaticity, for which new and important questions have been generated. We are looking forward for answers to these questions in the near future, since such ultranarrow AGNRs have been already synthesized in atomically precise form using bottom up approaches by two different groups. ${ }^{3-4}$

Methods. The theoretical and computational scheme used in the present investigation is based on real-space systematic DFT calculations on properly selected representative rectangular AGNRs of very small widths. The technical details have been described before. ${ }^{8-10,16}$ All geometrical structures have been optimized (with tight convergence criteria), using the hybrid PBE0 ${ }^{18}$ functional, employing the 6-31G(d) basis set. The complete set of calculations was performed with the GAUSSIAN program package. ${ }^{19}$ 


\section{TOC Graphic}

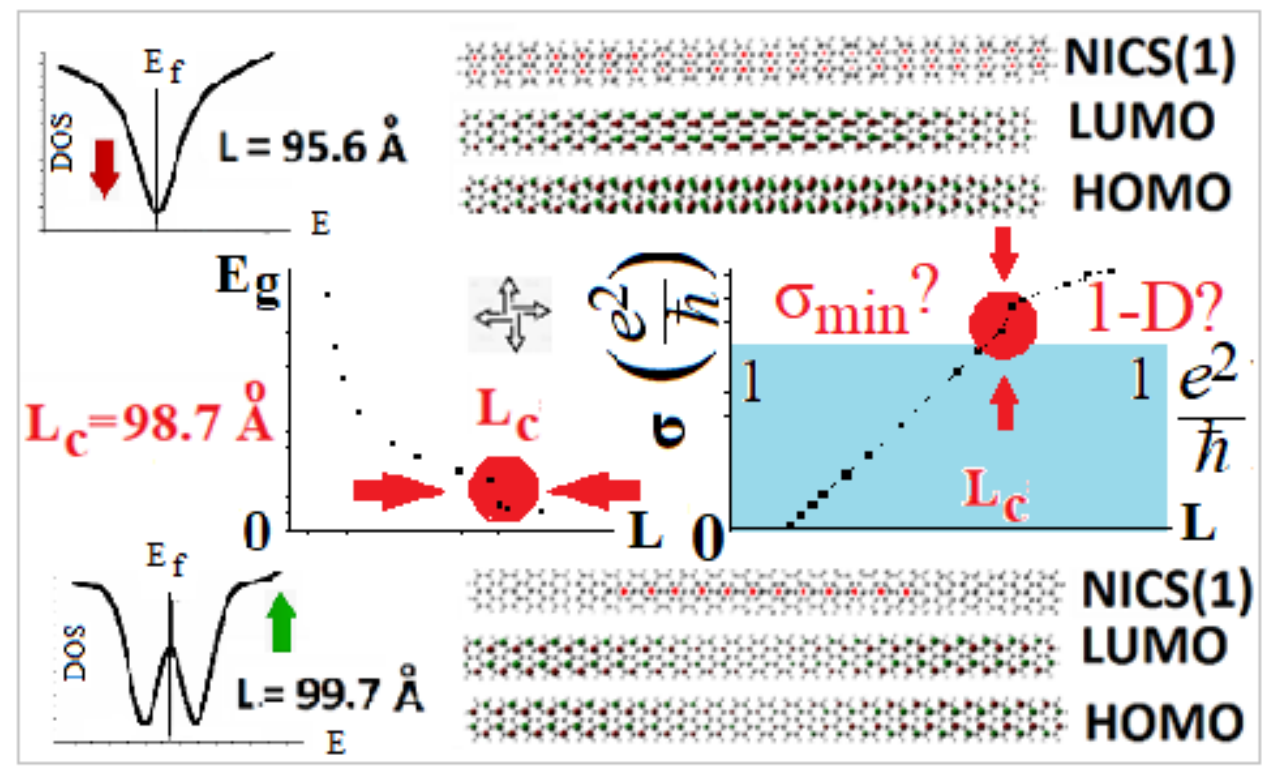

\section{References}


1) Segawa, Y.; Ito, H.; and Itami, K. Structurally uniform and atomically precise carbon nanostructures. Nat. Rev. Mats. 2016, 1, 15002-1-15002-14.

2) Cai, J.; Ruffieux,P.; Jaafar, R.;Bier,M.;Braun,T.; Blankenburg, S.; Muoth, M.; Seitsonen, A. P.;Saleh,M.; Feng,X.; et al. Atomically Precise Bottom-up Fabrication of Graphene Nanoribbons. Nature 2010, 466, 470-473.

3) Zhang, H.; Lin, H; Sun, K. et al. On-surface synthesis of rylene-type graphene nanoribbons. J. Am. Chem. Soc. 2015 137, 4022-4025.

4) Kimouche, A; Ervasti, M. M.; Drost, R.; Halonen, S.; Harju, A.; Joensuu, P. M.; Sainio, J.; and Liljeroth, P. Ultra-narrow metallic armchair graphene nanoribbons. Nature Comm. 2015, 6:10177, 1-6.

5) Ruffieux, P.; Cai, J.; Plumb, N. C.; Patthey, L.; Prezzi, D.; Ferretti, A.; Molinari, E.; Feng, X.; Müllen, K.; Pignedoli, C. A.; et al. Electronic Structure of Atomically Precise Graphene Nanoribbons. ACS Nano 2012, 6, 6930-6935.

6) Chen, Y-C; Oteyza, D. G.; Pedramrazi, Z; Chen, C.; Fischer, F. R.; Crommie, M. F. Tuning the Band Gap of Graphene Nanoribbons Synthesized from Molecular Precursors. ACS Nano. 2013, 6123-6128.

7) Talirz, L.; Söde, H; Dumslaff, T. et al. On-Surface Synthesis and Characterization of 9-Atom Wide Armchair Graphene Nanoribbons. ACS Nano. 2017, 11, 1380-1388.

8) Zdetsis, A. D.; and Economou, E.N. Rationalizing and Reconciling Energy Gaps and Quantum Confinement in Narrow Atomically Precise Armchair Graphene Nanoribbons. Carbon 2017, 116, 422-434.

9) Zdetsis, A. D. Bridging the Physics and Chemistry of Graphene(s): From Hückel's Aromaticity to Dirac's Cones and Topological Insulators. J. Phys. Chem A, 2020 $124,976-986$. 
10)Zdetsis, A. D. Do We Really Understand Graphene Nanoribbons? A New Understanding of the 3n, 3n \pm 1 Rule, Edge "Magnetism" and Much More. J. Phys. Chem. C 2020, 124, 7578-7584.

11) Novoselov, K. S.; Geim, A. K. ; Morozov, S. V. ; Jiang, D.; Katsnelson, M. I. et al. Two-dimensional gas of massless Dirac fermions in graphene, Nature 2005, 438, 197-200.

12) K. Ziegler K. Minimal conductivity of graphene: Nonuniversal values from the Kubo formula. Phys. Rev. B $\mathbf{2 0 0 7 ~ 7 5 , ~} 233407$.

13) Cho S. ; Fuhrer M. S. Charge Transport and Inhomogeneity near the Charge Neutrality Point in Graphene Phys. Rev B , 2008 77, 081402(R).

14) Economou, E.N. The Physics of Solids: Essentials and Beyond, Springer, 2010, (p. $519)$.

15) Schleyer, P.v.R.; Maerker, C.; Dransfeld, A.; Jiao, H.; van Eikema Hommes, N.J.R. Nucleus Independent Chemical Shifts: A simple and Efficient Aromaticity Probe, $J$. Am. Chem. Soc. 1996, 118, 6317-6318.

16) Zdetsis, A. D. Classics Illustrated: Clar's Sextet and Hückel's $(4 n+2) \pi$ Electron Rules. J. Phys. Chem. C, 2018, 122, 17526-17536.

17) Yang, L., Park, C.-H., Son, Y.-W., Cohen, M. L. \& Louie, S. G. Quasiparticle energies and band gaps in graphene nanoribbons. Phys. Rev. Lett. 2007, 99, 186801.

18) Adamo, C.; and Barone,V. Toward Reliable Density Functional Methods Without Adjustable Parameters: The PBE0 model. J. Chem. Phys., 1999, 110 6158-69.

19) Frisch, M. J.; Trucks, G.W. ; Schlegel, H. B.; Scuseria, G. E.; et al., Gaussian 09, Revision C.01, Gaussian, Inc., Wallingford CT, 2009. 